\title{
New Biological Dietary Feed Supplement for Laying Hens with Microelements Based on Duckweed (Lemna minor)
}

\author{
${ }^{1}$ Zuzanna Witkowska, ${ }^{1}$ Agnieszka Saeid, ${ }^{1}$ Katarzyna Chojnacka, ${ }^{2}$ Zbigniew Dobrzanski, \\ ${ }^{1}$ Henryk Gorecki, ${ }^{1}$ Izabela Michalak, ${ }^{2}$ Mariusz Korczynski and ${ }^{2}$ Sebastian Opalinski \\ ${ }^{1}$ Institute of Inorganic Technology and Mineral Fertilizers, \\ Faculty of Chemistry, Wrocław University of Technology, I-26, Poland \\ ${ }^{2}$ Department of Environment, \\ Wrocław University of Environmental and Life Sciences, Hygiene and Animal Welfare, Poland
}

Received 2012-11-29, Revised 2012-12-12; Accepted 2012-12-12

\begin{abstract}
In this study the applicability of enriched duckweed (Lemna minor) as a dietary supplement witch microelements is reported. In our previous studies, the technology of new feed additives with microelements based on duckweed biomass was elaborated. Here, we report the evaluation of the properties of a new product. The effect of duckweed enriched with microelements on the productivity parameters of laying hens was studied in zootechnical research. Birds feed was supplemented with duckweed enriched by biosorption process with microelements (Cu(II), Zn(II), $\mathrm{Co}(\mathrm{II}), \mathrm{Cr}(\mathrm{III}))$. In the feeding experiment, laying hens were divided into four experimental groups and one control group. The feeding experiment was conducted for 41 days. Samples of egg yolk, albumen, eggshells, blood, feathers and droppings were collected and the content of metal ions was determined by Inductively Coupled Plasma-Optical Emission Spectrometer with ultrasonic nebulizer. The amount of a given microelement transferred into the egg yolk and egg white was calculated. The eggshells thicknesses were measured with micrometer screw. The research showed that enriched Lemna minor improved the egg quality parameters. In all experimental groups, the increase of eggshell thickness was observed. In three of four experimental groups of hens, fed with diet containing biological form of microelements (Co(II), $\mathrm{Zn}(\mathrm{II}), \mathrm{Cr}$ (III)), the quantity of given microelement in the egg content increased. Therefore, the biosorption process can be applied not only for the supplementation of microelements in hens feed, but also to produce eggs biofortified with microelements-new functional food for human.
\end{abstract}

Keywords: Lemna Minor, Laying Hens, Biosorption, Feed Additives, Functional Food

\section{INTRODUCTION}

Biosorption, which uses the materials of biological origin, is metabolically independent process, which relies on the passive binding of microelements from aqueous solutions to the functional groups present on the cell surface of non-living biomass (Davis et al., 2003). Traditionally, biosorption is investigated as the process used in wastewater treatment. The mechanism of biosorption process has been described recently. Researchers have shown that biosorption occurs by several mechanisms, among which ion exchange dominates: the ions of alkali and alkaline earth metals, naturally bound with the functional groups on the surface of cells, are released into the solution and in their places other metal ions are bound (Chojnacka, 2010). Other mechanisms of biosorption that have much smaller contribution in the process, are microprecipitation, physical adsorption, complexation and chelation (Schiewer and Volesky, 1997).

Recently, a new perspective on the use of biosorption has been offered-not as a method for removing heavy metals from solutions, but as a method of biological materials enrichment in desirable elements (Chojnacka,

Correspondence Author: Zuzanna Witkowska, Wrocław University of Technology, Institute of Inorganic Technology and Mineral Fertilizers, Wrocław, Poland Tel: +48713203291 Fax: +48713203469 
$2007 ; 2010)$. Some of the elements play a role of micronutrients that are important to the health and growth, both for animals and for plants (i.e., zinc, copper, iron, chromium, manganese) (Gupta et al., 2008). The biological material edible to animals can be enriched with microelements in a simple process of biosorption and by that biological feed additives with microelements can be obtained (Chojnacka, 2010). Biosorption represents a potentially powerful tool to increase dietary intake of microelements in foods (Johns and Eyzaguirre, 2007) and it gives the opportunity to increase the content of microelements several fold, i.e., the content of $\mathrm{Cu}$ (II) in the biomass of macroalga Pithophora varia Wille after biosorption process increased 498 times (Michalak and Chojnacka, 2008), in the biomass of an aquatic plant Lemna minor (duckweed) 55 times (Chojnacka, 2006) and in the biomass of another aquatic freshwater plant Riccia fluitans 53 times (Chojnacka, 2007).

Nowadays, a special attention is being paid to the chemical form of microelements in animal diet. There has been a considerable interest in the application of chelated or organic form of microelements (i.e., zinc methionine, manganese methionine, iron methionine, zinc lysine, copper lysine) (Spears, 1996) instead of conventionally used mineral salts such as: sulfates (of manganese, copper, zinc, cobalt, iron), carbonates (of copper, zinc, cobalt), chlorides (of copper, zinc, cobalt) and oxides (of manganese, copper, zinc, iron) (McDowell, 1996). In the literature there are several reports, which showed the advantages of the organic over inorganic form of micronutrients (Dobrzanski et al., 2003; 2008; Spears, 1996), but on the other hand the main disadvantage of the organic form is high price (McDowell, 1996). Another option considers the application of the process of biosorption, whereby a biological material is enriched with microelements and used in animal diet, i.e., enriched by biosorption marine macroalgae-Enteromorpha sp. and Cladophora sp. (Michalak et al., 2011). In the literature it is reported that bioavailability of microelements from yeasts-Saccharomyces cerevisiae enriched with Se and $\mathrm{Zn}$ was higher than from inorganic forms such as sodium selenite and zinc oxide. Also, the selenium content in eggs from hens fed with enriched yeast was higher (10.4\%) in comparison with the eggs from hens fed with the diet supplemented with sodium selenite (Dobrzanski et al., 2003).

In this study, the application of organic form of microelements (Lemna minor enriched via biosorption process) as biological feed additive with microelements for laying hens is proposed. The main aim of the present work was to investigate the effect of biomass enriched with $\mathrm{Cu}$ (II), $\mathrm{Zn}$ (II), $\mathrm{Co}$ (II) and $\mathrm{Cr}$ (III) on the transfer of microelements to albumen, egg yolk, eggshell, blood, feathers and droppings. The choice of duckweed as a natural carrier of microelements in animal feed was justified by its good biosorption properties and also ubiquity. This aquatic plant is found floating on the surface of many ponds and slow-moving streams, as well as in rice paddies. Duckweed grows under a variety of climatic conditions in most parts of the world (Wahaab et al., 1995). Moreover, Lemna sp. with a natural property of binding metal ions from aqueous solutions, was found to be a very effective and cheap biosorbent (Chojnacka, 2006; Saadet et al., 2005) and bioaccumulator (Chojnacka, 2006; Wahaab et al., 1995; Prasad et al., 2001) of metal ions. Some attention was also paid to the application of duckweed in animal feeding. Previously it was shown that duckweed has the potential to be used as mineral feed additive in livestock nutrition (Chojnacka, 2006). Apart from high mineral content, duckweed is rich in protein (20$40 \%$ of dry weight), vitamins, carotenoids and fiber and can be successfully used as a soybean substitute in the feeding of laying hens and cattle (Hanczakowski et al., 1995; Haustein, 1990). In the literature it is reported that duckweed could replace a part of the traditional sources of protein (soybean and fish meal) (Men et al., 1997; Samnang, 1999) and that the supplementation of feed improved reproductive performance of sows (Men et al., 1997). It was also indicated that the feed supplementation with duckweed was much cheaper than the supplementation with ground soya beans and additionally introduced small amounts of fresh duckweed (30-40 $\mathrm{g} \mathrm{day}^{-1}$ ) to chickens diet, improved their growth rate (Samnang, 1999). Moreover, it was reported that addition of duckweed to laying hens diet significantly increased yolk pigmentation (Haustein, 1990). Of concern could be the content of toxic metals and microbial contamination. For this reason, chemical and microbiological tests are indispensable. Moyo et al. (2003) showed that Lemna minor is microbiologically safe.

The objective of the present work was to investigate the applicability of duckweed enriched with microelements by biosorption process as feed supplements with microelements for laying hens. This condensed form of essential elements could be used not only in the supplementation of deficiencies of microelements in animal diet, but also in the biofortification of eggs or meat with microelements. This kind of biofortified products could be applied as a new type of functional food, which would supply microelements not as inorganic salts, but in a highly bioavailable form.

\section{MATERIALS AND METHODS}

\subsection{Preparation of Biosorbent}

Aquatic plant Lemna minor was collected from the pond near Opatowek in Poland. Collected biomass was washed with tap water several times to remove foreign matter. 


\subsection{Biosorption Experiments}

Lemna minor biomass (Lm) was separately enriched with the following microelements: Cobalt (II) (Lm-Co), Chromium (III) (Lm-Cr), Copper (II) (Lm-Cu) and Zinc(II) (Lm-Zn) via biosorption. The enrichment processes were performed in $40 \mathrm{dm}^{3}$ containers with metal ion solutions in tap water at ambient temperature. The solutions were prepared by dissolving appropriate amounts of inorganic salts: $\mathrm{Co}\left(\mathrm{NO}_{3}\right)_{2} \cdot 6 \mathrm{H}_{2} \mathrm{O}, \mathrm{Cr}$ $\left(\mathrm{NO}_{3}\right)_{3} \cdot 9 \mathrm{H}_{2} \mathrm{O}, \quad \mathrm{CuSO}_{4} \cdot 5 \mathrm{H}_{2} \mathrm{O}, \quad \mathrm{ZnSO}_{4} \cdot 7 \mathrm{H}_{2} \mathrm{O} \quad(\mathrm{POCH}$, Gliwice, Poland) accepted as a mineral supplement for laying hens. The initial concentrations of metal ions in the solution were as follows: $5.77 .10^{-3} \mathrm{M}, 5.10 .10^{-3} \mathrm{M}$,

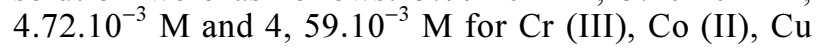
(II) and $\mathrm{Zn}(\mathrm{II})$, respectively. The biomass was exposed to microelement ions solutions for $4 \mathrm{~h}$. $\mathrm{pH}$ was adjusted with $\mathrm{NaOH} / \mathrm{HCl}$ (POCH, Gliwice, Poland) to 5. $\mathrm{pH}$ measurements were conducted with pH-meter equipped with an electrode InLab413 with the compensation of temperature (Mettler-Toledo, Seven Multi, Switzerland). The biomass concentration was $1.6 \%$ (16.3 $\mathrm{g}$ of fresh mass/L). Moisture of fresh plant biomass was evaluated as $94.3 \%$.

\subsection{Analytical Methods}

Multielemental analysis of the biomass was undertaken. The appropriate mass of biological samples $(0.1 \mathrm{~g}, 0.25 \mathrm{~g}, 0.5 \mathrm{~g}, 1.5$ and $2.5 \mathrm{~g}$ in the case of eggshells, droppings, feathers, albumen or egg yolk and blood, respectively) was digested in $5 \mathrm{~mL} 65 \% \mathrm{HNO}_{3}$ supra-pure grade (Merck, Germany) in Teflon vessels (microwave oven Milestone MLS-1200). After mineralization, all samples were diluted to $50 \mathrm{~mL}$. Inductively Coupled Plasma-Optical Emission Spectrometer with ultrasonic nebulizer (Varian VISTAMPX ICP-OES, Victoria, Australia) was used to determine the content of metal ions in fresh plant and in all digested and diluted biological samples in the Chemical Laboratory of Multielemental Analysis at Wrocław University of Technology, which is accredited by ILAC-MRA and Polish Centre for Accreditation ( $\mathrm{Nr}$ AB 969) (Michalak and Chojnacka, 2009).

To determine the eggshell thickness, micrometer screw (TSS Technical Services and Supplies, Germany) was used. The thickness of eggshell was measured in the central part of an eggshell, where the perimeter was the highest. The arithmetic mean values ( $\bar{x})$, Standard Deviations (SD) and significance of differences between the groups were examined with the use of computer software Statistica ver. 9.0. Normality of distribution was assessed by Shapiro-Wilk test. Data were found to be nonparametric, so the Tukey test was applied. Statistical significance between the groups was accepted for $\mathrm{p} \leq 0.05$.
Assuming that an egg is composed of yolk (26\%), albumen (60\%), eggshell (12\%), membrane (2\%), it was possible to calculate the mass of a given microelement $(\mathrm{mg})$, which was transferred to the albumen or yolk from the Equation 1:

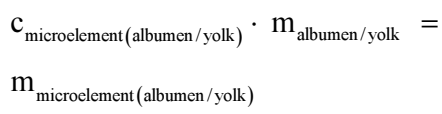

Where:

$\mathrm{c}_{\text {microelement(albumen/yolk) }}=$ The content of a given microelement in albumen or yolk $(\mathrm{mg} / \mathrm{kg})$

$\mathrm{m}_{\mathrm{albumen} / \mathrm{yolk}} \quad=$ The mass of albumen or yolk (kg)

$\mathrm{m}_{\text {microelement(albumen/yolk) }}=$ The mass of microelement in albumen or yolk (mg)

The Equation 2 was used to calculate the mass of microelement (mg), which was transferred from the feed to the egg content.

$$
\begin{aligned}
& \mathrm{c}_{\text {microelement(albumen) }} \cdot \mathrm{m}_{\text {albumen }}+\mathrm{c}_{\text {microelement }(\text { egg yolk })} \\
& \cdot \mathrm{m}_{\text {egg yolk }}=\mathrm{m}_{\text {microelement }(\mathrm{egg})}
\end{aligned}
$$

Where:

$\begin{aligned} \mathrm{c}_{\text {microelement(albumen })=} & \text { The content of a given } \\ & \text { microelement in albumen }(\mathrm{mg} / \mathrm{kg}) \\ \mathrm{m}_{\text {albumen }} & \text { The mass of albumen }(\mathrm{kg}) \\ \mathrm{c}_{\text {microelement(egg yolk })=} & \text { The content of a given } \\ & \text { microelement in egg yolk }(\mathrm{mg} / \mathrm{kg}) \\ \mathrm{m}_{\text {egg yolk }} & \text { The mass of egg yolk }(\mathrm{kg}) \\ \mathrm{m}_{\text {microelement(egg) }}= & \text { The mass of microelement in the } \\ & \text { egg }(\mathrm{mg})\end{aligned}$

\subsection{Feeding experiments}

\subsubsection{Feed}

Duckweed (Lemna minor) biomass was investigated as the biological carrier for microelements bound via biosorption process. Four experimental groups and one control group of laying hens were distinguished. The control group was fed with the standard feed (TASOMIX Company, Poland), which was composed of: corn, wheat, triticale, barley, soybean meal, sunflower meal, rape cake, feed fat, methionine hydroxy analog acid, $\mathrm{CaCO}_{3}$, $\mathrm{CaHPO}_{4} \cdot 2 \mathrm{H}_{2} \mathrm{O}, \mathrm{NaCl}, \mathrm{Na}_{2} \mathrm{SO}_{4}$. In the standard feed, all microelements were provided in the form of inorganic salts, with the exception of chromium, which is currently not added to feeds (AMARD, 2004; Jamroz, 2004). 
Table 1. The recommended content of microelements in feed and experimental supplementation of microelements

\begin{tabular}{lllc}
\hline $\begin{array}{l}\text { Lemna minor } \\
\text { enriched with } \\
\text { supplemented } \\
\text { microelement }\end{array}$ & $\begin{array}{l}\text { Requirement for } \\
\text { supplementation } \\
\text { of microelement } \\
(\mathrm{g} / \mathrm{kg} \text { of feed) }\end{array}$ & $\begin{array}{l}\text { Content of } \\
\text { microelement in } \\
\text { enriched Lemna } \\
\text { minor }(\mathrm{g} / \mathrm{kg})\end{array}$ & $\begin{array}{l}\text { Mass of enriched } \\
\text { Lemna minor } \\
\text { additive } \\
(\mathrm{mg} / \mathrm{kg} \mathrm{of} \mathrm{feed)}\end{array}$ \\
\hline $\mathrm{Lm}-\mathrm{Co}$ & $1^{*}$ & 42.862 & 0.0234 \\
$\mathrm{Lm}-\mathrm{Cu}$ & $10^{*}$ & 48.768 & 0.2060 \\
$\mathrm{Lm}-\mathrm{Cr}$ & $2^{*}$ & 49.207 & 0.0406 \\
$\mathrm{Lm}-\mathrm{Zn}$ & $50^{* *}$ & 60.067 & 0.8314 \\
\hline
\end{tabular}

*; Jamroz (2004) ${ }^{* * ;}$ (Smulikowska and Rutkowski, 2005)

Table 2. Mineral composition of natural Lemna minor and the standard feed

\begin{tabular}{|c|c|c|c|c|}
\hline Element & & $\begin{array}{l}\text { Natural Lemna minor } \\
\text { (mg/kg dry mass, } \\
\pm \mathrm{SD})\end{array}$ & $\begin{array}{l}\text { Standard feed } \\
\text { (mg/kg dry mass, } \\
\pm \mathrm{SD})\end{array}$ & $\begin{array}{l}\text { Maximum } \\
\text { content } \\
\text { in the feed }\end{array}$ \\
\hline \multirow[t]{6}{*}{ Microelements } & $\mathrm{Mn}$ & $333 \pm 49.9$ & $110 \pm 16.5$ & $150.00^{*}$ \\
\hline & $\mathrm{Zn}$ & $106 \pm 0.24$ & $89.6 \pm 13.4$ & $150.00^{*}$ \\
\hline & $\mathrm{Cu}$ & $5.82 \pm 0.873$ & $21.2 \pm 3.18$ & $25.00^{*}$ \\
\hline & Co & $1.68 \pm 0.423$ & $1.40 \pm 0.21$ & $2.00^{*}$ \\
\hline & $\mathrm{Fe}$ & $543 \pm 81.4$ & $247 \pm 37.0$ & $750.00^{*}$ \\
\hline & $\mathrm{Cr}$ & $26.5 \pm 3.97$ & $1.97 \pm 0.295$ & - \\
\hline \multirow{5}{*}{$\begin{array}{l}\text { Alkali and alkaline } \\
\text { earth metals }\end{array}$} & $\mathrm{K}$ & $10.6 \pm 1.59$ & $8080 \pm 1616$ & $0.25^{* *}$ \\
\hline & $\mathrm{Ca}$ & $11180 \pm 2236$ & $27021 \pm 5404$ & $3.50^{* *}$ \\
\hline & $\mathrm{Mg}$ & $2115 \pm 423$ & $2302 \pm 460$ & $0.05^{* *}$ \\
\hline & $\mathrm{Na}$ & $3260 \pm 3260$ & $1565 \pm 0.313$ & $0.15^{* *}$ \\
\hline & $\mathrm{Ba}$ & $30.8 \pm 4.62$ & $5.62 \pm 0.843$ & - \\
\hline \multirow[t]{3}{*}{ Toxic elements } & $\mathrm{Cd}$ & $<0.0045$ & $<0.0045$ & $1.00^{* * *}$ \\
\hline & $\mathrm{Ni}$ & $2.6 \pm 0.39$ & $0.801 \pm 0.12$ & - \\
\hline & $\mathrm{Pb}$ & $<0.0044$ & $5.07 \pm 0.760$ & $10.00^{* * *}$ \\
\hline \multirow[t]{2}{*}{ Other } & $\mathrm{Ti}$ & $4.98 \pm 2.49$ & $5.26 \pm 0.789$ & - \\
\hline & $\mathrm{Al}$ & $124 \pm 18.6$ & $221 \pm 33.15$ & - \\
\hline
\end{tabular}

*; mg/kg, AMARD (2004) ${ }^{* *} ; \%$, (Smulikowska and Rutkowski, 2005) ${ }^{* * *} ; \mathrm{mg} / \mathrm{kg}$, European (Commission, 2002)

The experimental groups were fed with the same feed, but a given microelement in the form of inorganic salt was replaced by Lemna minor enriched with this microelement by biosorption. In the case of $\mathrm{Lm}-\mathrm{Cr}$ group, the feed was supplemented with soybean meal enriched with $\mathrm{Cr}$ (III) ions and the standard feed remained unchanged since no chromium supplement was added to the standard feed. In experimental groups, supplementations with single microelement bound with the Lemna minor were undertaken. Remaining microelements were supplemented by inorganic salts (except of chromium). On the basis of the equilibrium biosorption capacity obtained from biosorption experiments the appropriate masses of enriched biomass of plant were evaluated (Table 1). Doses of micronutrients were established on the basis of legislation and nutritional recommendations (Table 1 and 2).

\subsection{Animals}

Laying hens (50 heads, 30 weeks old, Löhmann Brown) were divided into one control group and four experimental groups (10 hens per group) and were housed in pairs ( 5 replicates $(\mathrm{N})$ per group). The study was performed in three-tier battery system in the vivarium with controlled microclimate. Inside the vivarium the mean temperature was $20.8 \pm 1.45^{\circ} \mathrm{C}$, in the middle tier of the cages $21.4 \pm 1.80^{\circ} \mathrm{C}$ and outside the vivarium $15.7 \pm 1.45^{\circ} \mathrm{C}$. The mean relative humidity was $58.2 \pm 6.56 \%$ inside the vivarium, $62.4 \pm 7.17 \%$ in the middle tier of the cages and $58.8 \pm 9.73 \%$ outside the vivarium. Feed and water were available ad libitum.

\subsection{Sampling}

The feeding experiment was conducted for 41 days and was divided into three series of sampling: after 13 (series I), 27 (series II) and 41 (series III) days of 
feeding. After each series, all eggs from the experimental groups were weighed and the thickness of the eggshells was measured on the equator at 3 points in five randomly selected eggs from every cage. The mass of one egg was calculated as a quotient of mass of all eggs from given group per number of eggs. After series I and III, all hens from each group were weighed. Samples of albumen, egg yolk, eggshells, blood, feathers and droppings were collected at the end of the experiment. Blood and feathers were sampled from the vein under the wing from randomly selected hens from each group. Before blood sampling, heparin was added to the probe in order to prevent blood coagulation. Moreover, from all eggs collected after each series, albumens, yolks and eggshells were separated. Samples of albumens, yolks, eggshells, blood, droppings and feathers were homogenized. All samples, with the exception of eggshells, were kept in the freezer before multielemental analysis.

\section{RESULTS}

In Table 2 the mineral composition of natural biomass of Lemna minor is presented, as well as the mineral composition of standard feed used in these experiments. Table 3 presents the results of measurements of eggs masses and eggshell thickness and Fig. 1. presents the change of eggshell thickness during the experiment in a given group. Mineral content of samples taken during the feeding experiment is presented in Table 4 and 5, while Table 6 shows the amounts of microelements (mg) which were transferred to albumen or yolk from the feed, calculated according to Equation 1 and the amounts of microelements, which were transferred from the feed to the egg content (albumen and yolk), calculated according to Equation 2.

Table 3. Average mass of one egg $(\mathrm{g})$, the arithmetic mean $(\overline{\mathrm{x}})$ and Standard Deviation (SD) of eggshell thickness

\begin{tabular}{|c|c|c|c|c|}
\hline \multirow[b]{2}{*}{ Group } & \multicolumn{4}{|l|}{ Mass of egg $(\mathrm{g})$} \\
\hline & Series I & Series II & Series III & $\overline{\mathrm{x}} \pm \mathrm{SD}$ \\
\hline Control & 62.2 & 63 & 64.1 & $63.1 \pm 0.954$ \\
\hline $\mathrm{Lm}-\mathrm{Co}$ & 64.5 & 65 & 63.5 & $64.3 \pm 0.764$ \\
\hline $\mathrm{Lm}-\mathrm{Cr}$ & 61.9 & 62.5 & 63.8 & $62.7 \pm 1.015$ \\
\hline $\mathrm{Lm}-\mathrm{Cu}$ & 62.5 & 62.7 & 63 & $62.8 \pm 0.278$ \\
\hline $\mathrm{Lm}-\mathrm{Zn}$ & 63.9 & 64.1 & 61.8 & $63.3 \pm 1.274$ \\
\hline \multicolumn{5}{|c|}{ Mean eggshell thickness $( \pm S D, N=5, \mathrm{~mm})$} \\
\hline Group & Series I & Series II & Series III & $\overline{\mathrm{x}} \pm \mathrm{SD}$ \\
\hline Control & $0.391 \pm 0.340^{\mathrm{a}}$ & $0.416 \pm 0.032^{b, 1}$ & $0.484 \pm 0.071^{\mathrm{a}, \mathrm{b}, 3}$ & $0.430 \pm 0.046$ \\
\hline $\mathrm{Lm}-\mathrm{Co}$ & $0.417 \pm 0.047^{\mathrm{c}}$ & $0.450 \pm 0.048$ & $0.457 \pm 0.058^{\mathrm{c}}$ & $0.441 \pm 0.051$ \\
\hline $\mathrm{Lm}-\mathrm{Cr}$ & $0.421 \pm 0.070$ & $0.427 \pm 0.017$ & $0.437 \pm 0.041$ & $0.428 \pm 0.043$ \\
\hline $\mathrm{Lm}-\mathrm{Cu}$ & $0.383 \pm 0.032^{\mathrm{d}, \mathrm{e}}$ & $0.412 \pm 0.025^{\mathrm{d}, 2}$ & $0.414 \pm 0.048^{\mathrm{e}, 3}$ & $0.403 \pm 0.035$ \\
\hline $\mathrm{Lm}-\mathrm{Zn}$ & $0.399 \pm 0.038^{\mathrm{f}, \mathrm{g}}$ & $0.462 \pm 0.069^{f, 1,2}$ & $0.454 \pm 0.074^{\mathrm{g}}$ & $0.438 \pm 0.060$ \\
\hline
\end{tabular}

Letters ' $a$ ', ' $b$ ', ...: statistically significant differences between the series in one group, $p \leq 0.05$ Numbers ' 1 ', ' 2 ', ...: statistically significant differences between the groups in one series, $\mathrm{p} \leq 0.05$

Table 4. Mineral content of microelements, macroelements and toxic elements in egg yolk, albumen and eggshell

\begin{tabular}{|c|c|c|c|c|}
\hline \multirow[b]{2}{*}{ Element } & \multirow[b]{2}{*}{ Group } & \multicolumn{3}{|c|}{ Microelements ( $\pm \mathrm{SD}, \mathrm{N}=3, \mathrm{mg} / \mathrm{kg})$} \\
\hline & & Egg yolk & Albumen & Eggshell \\
\hline \multirow{5}{*}{$\overline{\mathrm{Co}}$} & Control & $\overline{L L D}<0.0009$ & 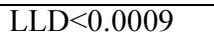 & LLD $<0.0009$ \\
\hline & Lm-Co & $0.0057 \pm 0.0014$ & LLD $<0.0009$ & $3.32 \pm 0.50$ \\
\hline & $\mathrm{Lm}-\mathrm{Cr}$ & $0.0083 \pm 0.0021$ & LLD $<0.0009$ & LLD $<0.0009$ \\
\hline & $\mathrm{Lm}-\mathrm{Cu}$ & $0.0095 \pm 0.0024$ & LLD $<0.0009$ & $0.8197 \pm 0.1230$ \\
\hline & Lm-Zn & $0.0076 \pm 0.0019$ & LLD $<0.0009$ & $2.14 \pm 0.32$ \\
\hline \multirow[t]{5}{*}{$\mathrm{Cr}$} & Control & $0.0731 \pm 0.0183$ & LLD $<0.00035$ & $0.327 \pm 0.477$ \\
\hline & Lm-Co & $0.118 \pm 0.018$ & LLD $<0.00035$ & $2.48 \pm 0.37$ \\
\hline & $\mathrm{Lm}-\mathrm{Cr}$ & $0.0779 \pm 0.0195$ & LLD $<0.00035$ & LLD $<0.00035$ \\
\hline & $\mathrm{Lm}-\mathrm{Cu}$ & $0.0678 \pm 0.0169$ & LLD $<0.00035$ & $0.242 \pm 0.036$ \\
\hline & Lm-Zn & $0.0790 \pm 0.0197$ & $\mathrm{LLD}<0.00035$ & LLD $<0.00035$ \\
\hline \multirow[t]{5}{*}{$\mathrm{Cu}$} & Control & $1.66 \pm 0.25$ & $0.250 \pm 0.037$ & $3.83 \pm 0.57$ \\
\hline & Lm-Co & $1.74 \pm 0.26$ & $0.299 \pm 0.045$ & $1.77 \pm 0.27$ \\
\hline & $\mathrm{Lm}-\mathrm{Cr}$ & $1.79 \pm 0.27$ & $0.322 \pm 0.048$ & $2.93 \pm 0.44$ \\
\hline & $\mathrm{Lm}-\mathrm{Cu}$ & $1.44 \pm 0.22$ & $0.331 \pm 0.050$ & $3.51 \pm 0.53$ \\
\hline & Lm-Zn & $1.68 \pm 0.25$ & $0.309 \pm 0.077$ & $1.15 \pm 0.17$ \\
\hline \multirow[t]{3}{*}{$\mathrm{Mn}$} & Control & $0.694 \pm 0.104$ & $0.0106 \pm 0.0027$ & $0.9015 \pm 0.1350$ \\
\hline & Lm-Co & $0.686 \pm 0.103$ & $0.0220 \pm 0.0055$ & $1.46 \pm 0.22$ \\
\hline & $\mathrm{Lm}-\mathrm{Cr}$ & $0.834 \pm 0.125$ & $0.0137 \pm 0.0034$ & $1.71 \pm 0.26$ \\
\hline
\end{tabular}


Table 4. Continued

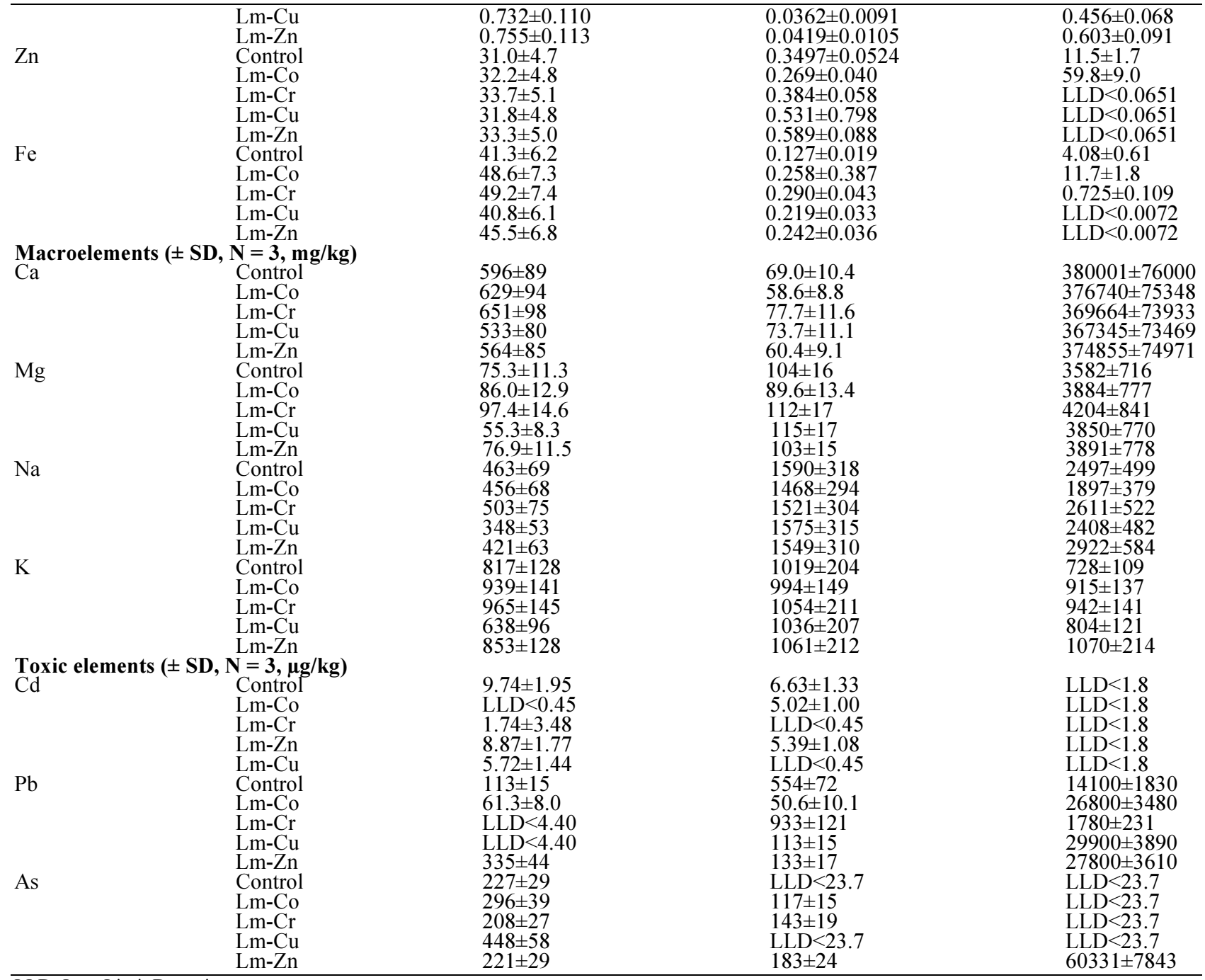

LLD; Low Limit Detection

Table 5. Mineral content of microelements, macroelements and toxic elements in blood, feathers and droppings

\begin{tabular}{|c|c|c|c|c|}
\hline \multirow[b]{2}{*}{ Element } & \multicolumn{4}{|c|}{ Microelements ( $\pm \mathrm{SD}, \mathrm{N}=3, \mathrm{mg} / \mathrm{kg})$} \\
\hline & Group & Blood & Feathers & Droppings \\
\hline \multirow[t]{5}{*}{$\overline{\mathrm{Co}}$} & Control & $0.0077 \pm 0.0019$ & $0.0783 \pm 0.0196$ & $0.311 \pm 0.047$ \\
\hline & Lm-Co & $0.0043 \pm 0.0011$ & $0.0725 \pm 0.0181$ & $0.339 \pm 0.051$ \\
\hline & $\mathrm{Lm}-\mathrm{Cr}$ & $0.0078 \pm 0.0019$ & $0.1638 \pm 0.0246$ & $0.709 \pm 0.106$ \\
\hline & $\mathrm{Lm}-\mathrm{Cu}$ & $0.0076 \pm 0.0019$ & $0.1386 \pm 0.0208$ & $0.492 \pm 0.074$ \\
\hline & Lm-Zn & LLD $<0.0009$ & $0.1088 \pm 0.0163$ & $0.447 \pm 0.067$ \\
\hline \multirow[t]{5}{*}{$\mathrm{Cr}$} & Control & $0.0664 \pm 0.0166$ & $0.182 \pm 0.027$ & $0.271 \pm 0.041$ \\
\hline & Lm-Co & $0.0665 \pm 0.0166$ & $0.142 \pm 0.021$ & $0.290 \pm 0.043$ \\
\hline & $\mathrm{Lm}-\mathrm{Cr}$ & $0.0644 \pm 0.0161$ & $0.315 \pm 0.048$ & $0.314 \pm 0.047$ \\
\hline & $\mathrm{Lm}-\mathrm{Cu}$ & $0.0608 \pm 0.015$ & $0.00042 \pm 0.00053$ & $0.258 \pm 0.039$ \\
\hline & Lm-Zn & $0.0679 \pm 0.0169$ & $0.2590 \pm 0.0388$ & $0.883 \pm 0.132$ \\
\hline \multirow[t]{2}{*}{$\mathrm{Cu}$} & Control & $0.4602 \pm 0.0690$ & $4.052 \pm 0.608$ & $13.3 \pm 2.0$ \\
\hline & $\mathrm{Lm}-\mathrm{Co}$ & $0.453 \pm 0.068$ & $5.75 \pm 0.86$ & $20.6 \pm 3.1$ \\
\hline
\end{tabular}


Table 5. Continued

\begin{tabular}{|c|c|c|c|c|}
\hline \multicolumn{5}{|c|}{$0.401 \pm 0.060$} \\
\hline & $\mathrm{Lm}-\mathrm{Cu}$ & $0.428 \pm 0.064$ & $3.76 \pm 0.56$ & $14.9 \pm 2.2$ \\
\hline & Lm-Zn & $0.461 \pm 0.069$ & $5.27 \pm 0.79$ & $25.2 \pm 3.8$ \\
\hline \multirow[t]{5}{*}{$\mathrm{Mn}$} & Control & $0.138 \pm 0.021$ & $8.1337 \pm 1.2200$ & $68.8 \pm 10.3$ \\
\hline & Lm-Co & $0.154 \pm 0.023$ & $11.4577 \pm 1.7200$ & $81.9 \pm 12.3$ \\
\hline & $\mathrm{Lm}-\mathrm{Cr}$ & $0.167 \pm 0.025$ & $16.438 \pm 2.470$ & $108.9 \pm 16.3$ \\
\hline & $\mathrm{Lm}-\mathrm{Cu}$ & $0.174 \pm 0.021$ & $12.9118 \pm 1.9400$ & $89.3 \pm 13.4$ \\
\hline & Lm-Zn & $0.194 \pm 0.029$ & $11.3774 \pm 1.7100$ & $87.4 \pm 13.1$ \\
\hline \multirow[t]{5}{*}{$\mathrm{Zn}$} & Control & $7.622 \pm 1.140$ & $245 \pm 37$ & $71.2 \pm 10.7$ \\
\hline & $\mathrm{Lm}-\mathrm{Co}$ & $6.48 \pm 0.97$ & $292 \pm 44$ & $69.8 \pm 10.5$ \\
\hline & $\mathrm{Lm}-\mathrm{Cr}$ & $6.99 \pm 1.05$ & $356 \pm 38$ & $60.5 \pm 9.1$ \\
\hline & $\mathrm{Lm}-\mathrm{Cu}$ & $7.55 \pm 1.13$ & $259 \pm 39$ & $93.9 \pm 14.1$ \\
\hline & Lm-Zn & $7.83 \pm 1.17$ & $251 \pm 38$ & $108 \pm 16$ \\
\hline \multirow[t]{5}{*}{$\mathrm{Fe}$} & Control & $251 \pm 38$ & $39.9 \pm 6.0$ & $182 \pm 27$ \\
\hline & Lm-Co & $233 \pm 35$ & $40.0 \pm 6.0$ & $123 \pm 18$ \\
\hline & $\mathrm{Lm}-\mathrm{Cr}$ & $252 \pm 38$ & $47.6 \pm 7.1$ & $191 \pm 29$ \\
\hline & $\mathrm{Lm}-\mathrm{Cu}$ & $247 \pm 37$ & $32.2 \pm 4.8$ & $137.9 \pm 20.7$ \\
\hline & Lm-Zn & $246 \pm 37$ & $47.8 \pm 7.2$ & $130.6 \pm 19.6$ \\
\hline \multicolumn{5}{|c|}{ Macroelements $( \pm \mathrm{SD}, \mathrm{N}=3, \mathrm{mg} / \mathrm{kg})$} \\
\hline \multirow[t]{5}{*}{$\mathrm{Ca}$} & Control & $138 \pm 21$ & $1317 \pm 263$ & $5902 \pm 1180$ \\
\hline & Lm-Co & $152 \pm 23$ & $1199 \pm 240$ & $4797 \pm 959$ \\
\hline & $\mathrm{Lm}-\mathrm{Cr}$ & $135 \pm 20$ & $1837 \pm 367$ & $7356 \pm 1471$ \\
\hline & $\mathrm{Lm}-\mathrm{Cu}$ & $135 \pm 20$ & $1256 \pm 251$ & $4051 \pm 810$ \\
\hline & Lm-Zn & $153 \pm 23$ & $1436 \pm 287$ & $4372 \pm 874$ \\
\hline \multirow[t]{5}{*}{$\mathrm{Mg}$} & Control & $38.9 \pm 5.8$ & $189 \pm 28$ & $1150 \pm 230$ \\
\hline & Lm-Co & $39.1 \pm 5.9$ & $183 \pm 27$ & $1489 \pm 298$ \\
\hline & $\mathrm{Lm}-\mathrm{Cr}$ & $36.9 \pm 5.5$ & $249 \pm 37$ & $1892 \pm 378$ \\
\hline & $\mathrm{Lm}-\mathrm{Cu}$ & $37.8 \pm 5.7$ & $149 \pm 22$ & $1424 \pm 285$ \\
\hline & $\mathrm{Lm}-\mathrm{Zn}$ & $42.6 \pm 6.4$ & $167 \pm 25$ & $1212 \pm 242$ \\
\hline \multirow[t]{5}{*}{$\mathrm{Na}$} & Control & $1925 \pm 385$ & $2002 \pm 400$ & $862 \pm 129$ \\
\hline & Lm-Co & $1971 \pm 394$ & $1337 \pm 267$ & $737 \pm 110$ \\
\hline & $\mathrm{Lm}-\mathrm{Cr}$ & $1846 \pm 369$ & $1472 \pm 294$ & $1026 \pm 205$ \\
\hline & $\mathrm{Lm}-\mathrm{Cu}$ & $1894 \pm 379$ & $1188 \pm 237$ & $1098 \pm 219$ \\
\hline & Lm-Zn & $1899 \pm 380$ & $1660 \pm 332$ & $773 \pm 116$ \\
\hline \multirow[t]{5}{*}{$\mathrm{K}$} & Control & $902 \pm 135$ & $721 \pm 108$ & $4715 \pm 943$ \\
\hline & $\mathrm{Lm}-\mathrm{Co}$ & $895 \pm 134$ & $970 \pm 145$ & $5138 \pm 1028$ \\
\hline & Lm-Cr & $873 \pm 130$ & $1309 \pm 262$ & $5218 \pm 1043$ \\
\hline & $\mathrm{Lm}-\mathrm{Cu}$ & $897 \pm 134$ & $864 \pm 130$ & $4676 \pm 935$ \\
\hline & $\mathrm{Lm}-\mathrm{Zn}$ & $913 \pm 137$ & $1072 \pm 214$ & $4386 \pm 877$ \\
\hline \multicolumn{5}{|c|}{ Toxic elements $( \pm \mathrm{SD}, \mathrm{N}=3, \mu \mathrm{g} / \mathrm{kg})$} \\
\hline \multirow[t]{5}{*}{$\mathrm{Cd}$} & Control & $33.9 \pm 6.8$ & $17.5 \pm 3.5$ & $79.0 \pm 15.8$ \\
\hline & Lm-Co & $31.8 \pm 6.4$ & $11.0 \pm 2.2$ & $94.7 \pm 18.9$ \\
\hline & $\mathrm{Lm}-\mathrm{Cr}$ & $30.7 \pm 6.1$ & $22.0 \pm 4.4$ & $143 \pm 19$ \\
\hline & $\mathrm{Lm}-\mathrm{Zn}$ & $34.9 \pm 7.0$ & $39.6 \pm 7.9$ & $83.2 \pm 16.6$ \\
\hline & $\mathrm{Lm}-\mathrm{Cu}$ & $35.3 \pm 7.1$ & $\mathrm{LLD}<0.45$ & $84.2 \pm 16.8$ \\
\hline \multirow[t]{5}{*}{$\mathrm{Pb}$} & Control & $229 \pm 30$ & $939 \pm 122$ & $362 \pm 47$ \\
\hline & $\mathrm{Lm}-\mathrm{Co}$ & $219 \pm 29$ & $1320 \pm 172$ & $469 \pm 61$ \\
\hline & $\mathrm{Lm}-\mathrm{Cr}$ & $109 \pm 14$ & $907 \pm 118$ & $607 \pm 78.9$ \\
\hline & $\mathrm{Lm}-\mathrm{Cu}$ & $92.4 \pm 18.5$ & $617 \pm 80$ & $1450 \pm 188$ \\
\hline & $\mathrm{Lm}-\mathrm{Zn}$ & $181 \pm 23$ & $713 \pm 93$ & $601 \pm 78$ \\
\hline \multirow[t]{5}{*}{ As } & Control & $129 \pm 17$ & $1300 \pm 169$ & $1040 \pm 135$ \\
\hline & Lm-Co & $194 \pm 25$ & $924 \pm 120$ & $1120 \pm 146$ \\
\hline & $\mathrm{Lm}-\mathrm{Cr}$ & $133 \pm 17$ & $1045 \pm 136$ & LLD $<23.7$ \\
\hline & $\mathrm{Lm}-\mathrm{Cu}$ & $116 \pm 15$ & $878 \pm 114$ & $1630 \pm 212$ \\
\hline & $\mathrm{Lm}-\mathrm{Zn}$ & $124 \pm 16$ & $678 \pm 88$ & $957 \pm 124$ \\
\hline
\end{tabular}


Zuzanna Witkowska et al. / American Journal of Agricultural and Biological Sciences 7 (4) (2012) 482-493

Table 6. The amount of microelements in albumen, yolk and in the egg content (albumen + yolk)

The amount of microelements

\begin{tabular}{|c|c|c|c|c|c|c|c|}
\hline Microelement & Group & Yolk (mg) & $\begin{array}{l}\text { Increase }(\uparrow) / \\
\operatorname{Decrease}(\downarrow)(\%)\end{array}$ & $\begin{array}{l}\text { Albumen } \\
(\mathrm{mg})\end{array}$ & $\begin{array}{l}\text { Increase }(\uparrow) / \\
\text { Decrease }(\downarrow)(\%)\end{array}$ & $\begin{array}{l}\text { Egg content } \\
(\mathrm{mg})\end{array}$ & $\begin{array}{l}\text { Increase }(\uparrow) / \\
\text { Decrease }(\downarrow)(\%)\end{array}$ \\
\hline \multirow[t]{2}{*}{$\mathrm{Co}(\mathrm{II})$} & Control & $<1.50 \cdot 10^{-5}$ & $>527(\uparrow)$ & $<3.46 \cdot 10^{-5}$ & $>0.867$ (â) & $<5.0 \cdot 10^{-5}$ & $>156(\uparrow)$ \\
\hline & Lm-Co & $9.41 \cdot 10^{-5}$ & & $<3.43 \cdot 10^{-5}$ & & $<1.28 \cdot 10^{-4}$ & \\
\hline \multirow[t]{2}{*}{$\mathrm{Cr}(\mathrm{III})$} & Control & $1.22 \cdot 10^{-3}$ & $5.73(\uparrow)$ & $<1.34 \cdot 10^{-5}$ & - & $<1.23 \cdot 10^{-3}$ & $>6.50(\uparrow)$ \\
\hline & $\mathrm{Lm}-\mathrm{Cr}$ & $1.29 \cdot 10^{-3}$ & & $<1.34 \cdot 10^{-5}$ & & $<1.31 \cdot 10^{-3}$ & \\
\hline \multirow[t]{2}{*}{$\mathrm{Cu}(\mathrm{II})$} & Control & 0.0277 & $14.8(\downarrow)$ & 0.01 & $30.0(\uparrow)$ & 0.037 & $3.22(\downarrow)$ \\
\hline & $\mathrm{Lm}-\mathrm{Cu}$ & 0.0236 & & 0.013 & & 0.036 & \\
\hline \multirow[t]{2}{*}{$\mathrm{Zn}(\mathrm{II})$} & Control & 0.517 & $3.48(\downarrow)$ & 0.013 & $69.2(\uparrow)$ & 0.53 & $5.01(\uparrow)$ \\
\hline & $\mathrm{Lm}-\mathrm{Zn}$ & 0.535 & & 0.022 & & 0.557 & \\
\hline
\end{tabular}

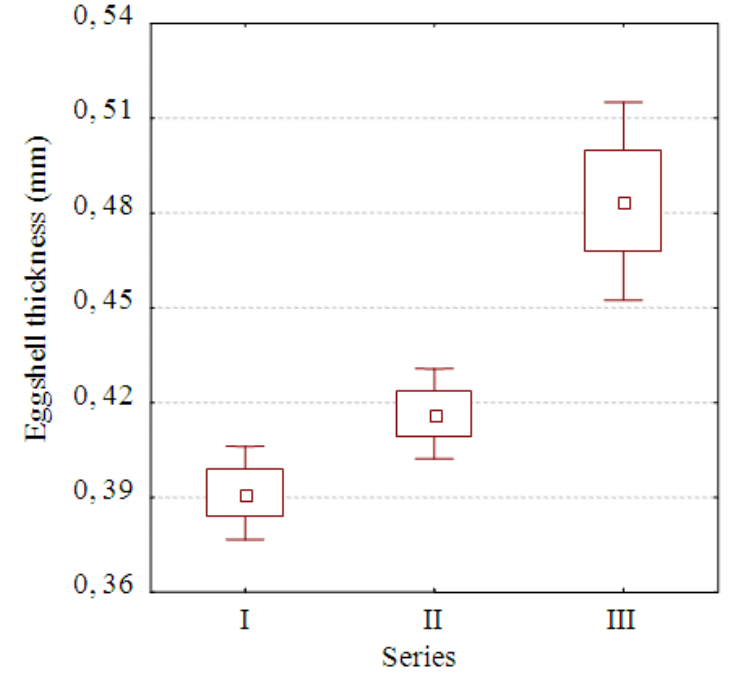

(a)

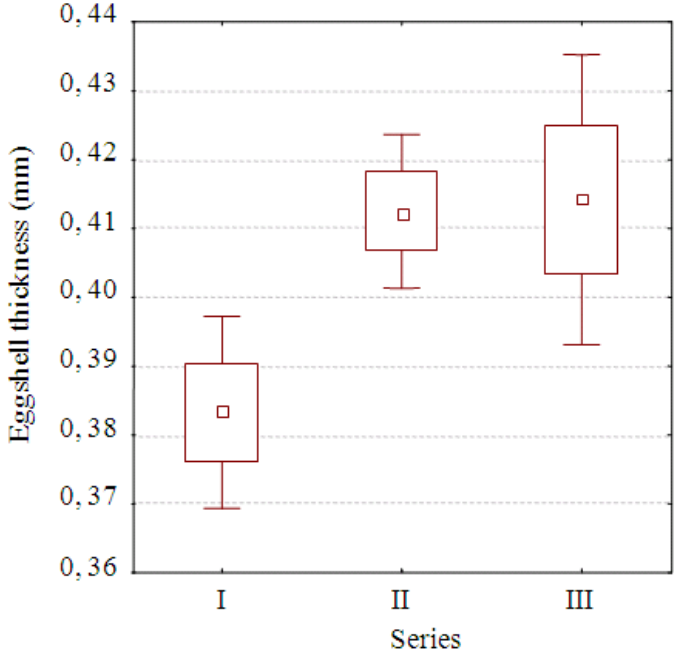

(b)

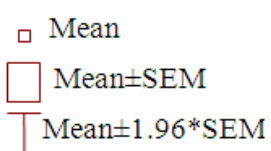

Mean $\pm 1.96 *$ SEM

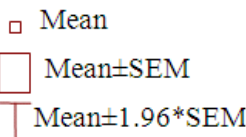


Zuzanna Witkowska et al. / American Journal of Agricultural and Biological Sciences 7 (4) (2012) 482-493
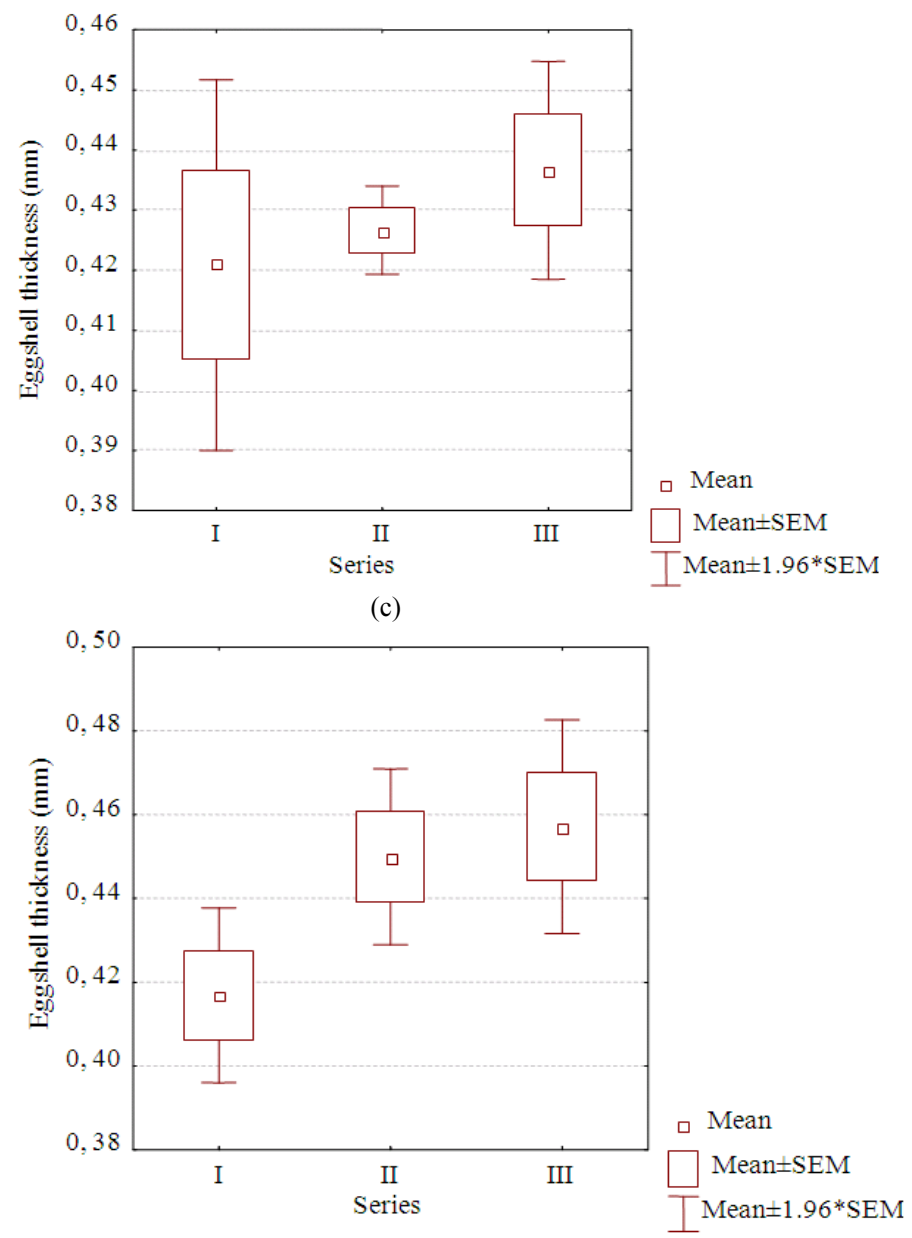

(d)

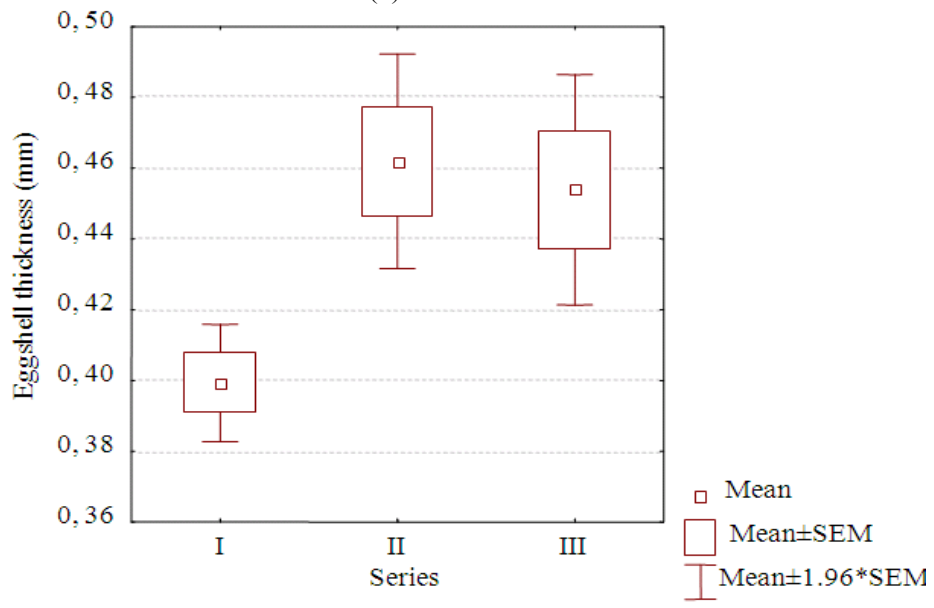

(e)

Fig. 1. Eggshell thickness during the experiment for control and experimental groups: (a) control group, (b) Lm-Cu group, (c) Lm-Cr group, (d) Lm-Co group, (e) Lm-Zn group 


\section{DISCUSSION}

Previously, new technology of biological feed supplements with microelements bound to the biomass of Lemna minor was elaborated (Chojnacka, 2006). The bioresource was found to be effective in concentrating microelement ions from the solution (i.e., over 68, 127 and 534 times for $\mathrm{Cr}$ (III), $\mathrm{Zn}$ (II) and $\mathrm{Cu}$ (II), respectively) by ions exchange mechanism. Cations were bound to carboxyl groups present on the surface of cells. In the present work utilitarian properties of new biological dietary supplement with microelements were investigated in zootechnical studies. Bioavailability of microelements (zinc, iron, copper and chromium) bound with the biomass of duckweed for laying hens was tested and compared with an inorganic dietary supplements. It was hypothesized that biological form of microelements whereby microelements are bound, similarly as in chelates, to carboxyl group should be a valuable and cost-effective alternative.

Here we report the effect of supplementation of feed with enriched aquatic plant on hens body weight, eggshell thickness and mineral content of albumen, egg yolk, eggshells, blood, feathers and droppings. The known amount of a given microelement bound with Lemna minor was supplemented and its level was determined in the collected samples using multielemental analysis to assess the influence of this new kind of mineral feed additive on health condition of animals.

The tested material was almost free of toxic metals (Table 2), their levels were below the restricted levels accepted by the obligatory law (DPMARD, 2012). Content of both lead and cadmium in natural Lemna minor was very low (below the lower detection limit). In many cases, the content of elements was higher than in the standard feed (Mn, Zn, Co, Fe, Cr, K, Na, Ba).

\subsection{The effect of Supplementation of Enriched with Microelements Lemna Minor on Egg Quality Parameters and Laying Hens Body Weight}

The increase of the mass of eggs was observed in the case of two experimental groups and the control group (Table 3). The highest egg mass increase was noticed in the $\mathrm{Lm}-\mathrm{Cr}$ group, by $3.12 \%$. In the control group the increase by $2.96 \%$ was noticed. The average weight of eggs from groups fed with Lm-Co and Lm-Zn slightly decreased, 1.55 and $3.21 \%$ respectively. There was no statistically significant difference in the weight of hens, which was measured at the beginning and at the end of the experiment.

The increase of eggshell thickness increase was observed in the case of all groups, both experimental and control. The highest increase of eggshell thickness during the experiment (between series I and III) was observed for the control group, by $28.3 \%$. In the experimental groups the highest increase was found for the Lm-Zn group, by $13.8 \%$. For $\mathrm{Lm}-\mathrm{Co}, \mathrm{Lm}-\mathrm{Cu}$ and $\mathrm{Lm}-\mathrm{Cr}$ the eggshell thickness increase was by $9.59,8.09$ and $3.80 \%$, respectively. The mean eggshell thickness was the highest in the Lm-Co group, higher by $2.33 \%$ in comparison with the mean eggshell thickness of the control group.

It was observed that in the case of all experimental groups, except for Lm-Cr, the highest increase of eggshell thickness was observed between the series I and II, while for the control group the highest increase was observed between the series II and III (Fig. 1). The strongest trend can be observed for $\mathrm{Lm}-\mathrm{Cu}$ and $\mathrm{Lm}-\mathrm{Zn}$ group, where statistically significant differences $(\mathrm{p} \leq$ 0.05 ) between the series I and II were found. For example, in $\mathrm{Lm}-\mathrm{Cu}$ group, the eggshell thickness increase between the series I and II was $7.57 \%$ and between the series II and III only $0.485 \%$. In the control group the eggshell thickness increase between the series I and II was found $6.4 \%$, while between the series II and III by $16.3 \%$. Also, in the series II statistically significant differences were observed in eggshell thickness between the control group and the groups of $\mathrm{Lm}-\mathrm{Zn}$ and $\mathrm{Lm}-\mathrm{Cu}$. This can indicate that eggshells from hens fed with biological supplement became thicker sooner than eggshells from the hens fed with the feed with inorganic salts.

\subsection{The Influence of Supplemented Microelements on their Content in Eggs}

In Lm-Co group the content of Co in egg yolk was $533 \%$ higher in comparison with the control group (Table 4). In eggshells, significant increase of Co content was observed, over 3500 times. Considering $\mathrm{Cr}$ content in collected samples in the Lm-Cr group it can be seen that the duckweed supplement increased $\mathrm{Cr}$ content in egg yolk by $6.57 \%$. In eggshells the content of $\mathrm{Cr}$ was found lower by over $99.2 \%$. In $\mathrm{Lm}-\mathrm{Cu}$ group the content of $\mathrm{Cu}$ in albumen was $32.4 \%$ higher. In the Lm-Zn group, Zn content in egg yolks was $7.4 \%$ higher. Also, in albumen the increase of $\mathrm{Zn}$ was found, $68.4 \%$. In eggshells, Zn content significantly decreased, over $99.9 \%$, in comparison with the control group.

Supplementation of feed with enriched Lemna minor increased the amount of given microelement in albumen and yolk. Supplements increased $\mathrm{Co}$ and $\mathrm{Cr}$ amounts in egg yolk, $\mathrm{Cu}$ amount in albumen and $\mathrm{Zn}$ amount in albumen and egg yolk. The highest increase of the total amount of given microelement in the egg content was found in Lm-Co group, 156\%. 


\subsection{The Influence of Supplemented Microelements on Their Content in Blood, Feathers and Droppings}

In droppings in every experimental group the content of microelement supplemented in biological form was higher than in the control group: 9.0, 12.015 .9 and $51.7 \%$ in the $\mathrm{Lm}-\mathrm{Co}, \mathrm{Lm}-\mathrm{Cu}, \mathrm{Lm}-\mathrm{Cr}$ and $\mathrm{Lm}-\mathrm{Zn}$ group, respectively (Table 5). In the case of $\mathrm{Lm}-\mathrm{Co}$ and $\mathrm{Lm}-\mathrm{Cu}$ groups the content of supplemented microelement in blood and feather samples was lower than in the control group. In Lm-Zn group the increase of $\mathrm{Zn}$ content in feathers and blood was observed, while in the $\mathrm{Lm}-\mathrm{Cr}$ group the $\mathrm{Cr}$ content in blood remained unchanged and in feathers the increase by $73.1 \%$ was found.

\subsection{Interactions (Antagonism and Synergism) Between Elements}

Synergism interactions were observed between all experimental groups.

\subsection{Other Microelements}

The increase of Mn content was observed in all albumen samples. The highest increase was for $\mathrm{Lm}-\mathrm{Zn}$ group, $295 \%$. In egg yolks the increase of Mn was found for every experimental group with the exception of LmCo group. The highest increase was observed for $\mathrm{Lm}-\mathrm{Cr}$ group, $20.2 \%$. The Mn content in droppings, feathers and blood was higher in every experimental group in comparison with the control group.

Fe content in albumen increased in all experimental groups. In comparison with the control group. Fe content was higher by at least $72.4 \%$ in the case of $\mathrm{Lm}-\mathrm{Cu}$, the highest increase was in sample taken from $\mathrm{Lm}-\mathrm{Cr}$ group, by $128 \%$. The increase was also observed in egg yolks with the exception of samples from $\mathrm{Lm}-\mathrm{Cu}$ group. The highest increase was found in sample from Lm-Cr group, $19.1 \%$. In blood and in droppings $\mathrm{Fe}$ content was lower in every experimental group except $\mathrm{Lm}-\mathrm{Cr}$ group.

\subsection{Macroelements}

In all experimental groups slight increase of magnesium content and slight decrease of $\mathrm{Ca}$ content in eggshells was observed. $\mathrm{Na}$ content was lower in comparison with the control group in albumen and feathers in all experimental groups. $\mathrm{K}$ content in eggshells and feathers was higher in comparison with the control group in all experimental groups.

\subsection{Toxic Metal Ions}

Cd was excreted with droppings in higher amounts in all experimental groups when compared with the control group. Both in egg yolks and albumen, Cd content was lower in all experimental groups. In egg yolks the highest decrease was observed for Lm-Co group, at least by $95.4 \%$, in albumen the lowest content of cadmium were found in $\mathrm{Lm}-\mathrm{Cr}$ and $\mathrm{Lm}-\mathrm{Cu}$ groups, at least by $93.2 \%$ lower in comparison with the control group. $\mathrm{Pb}$ content in droppings in all experimental groups was higher, in samples from $\mathrm{Lm}-\mathrm{Cu}$ group the increase of $\mathrm{Pb}$ was observed by $300 \%$. The $\mathrm{Pb}$ content was lower when compared with the control group in blood samples in all experimental groups. It can be concluded that feed supplementation with enriched Lemna minor resulted in cadmium and lead excretion into droppings and causes the decrease of $\mathrm{Cd}$ content in eggs and $\mathrm{Pb}$ content in blood.

\section{CONCLUSION}

In this research the applicability of duckweed enriched with microelements by biosorption process as the feed supplement for laying hens was investigated. Enriched Lemna minor improved egg quality parameters. The eggshell thickness increased in all experimental groups. The total amount of microelements in the egg content increased in three of four experimental groups. The new biological supplement increased Co content in egg yolk and eggshells, $\mathrm{Cr}$ content in egg yolk and also $\mathrm{Cu}$ and $\mathrm{Zn}$ content in albumen and yolk. In all experimental groups the $\mathrm{Cd}$ content in egg yolk and albumen samples was lower, as well as the $\mathrm{Pb}$ content in blood samples. Therefore, biological form of microelements could be a valuable and cost-effective alternative to inorganic dietary supplements and expensive chelates. Moreover, enriched duckweed could be used not only in animal diet supplementation, but also in the biofortification of eggs with microelements-new functional food for human.

\section{ACKNOWLEDGEMENT}

The study was granted by the National Centre for Research and Development nr NR05-0014-10

\section{REFERENCES}

AMARD, 2004. Lists of feed additives and feed materials.

Chojnacka, K., 2006. The application of multielemental analysis in the elaboration of technology of mineral feed additives based on Lemna minor biomass. Talanta, 70: 966-972. PMID: 18970868 
Chojnacka, K., 2007. Biosorption and bioaccumulation of microelements by Riccia fluitans in single and multi-metal system. Bioreso. Technol., 98: 29192925. DOI: 10.1016/j.biortech.2006.10.001

Chojnacka, K., 2010. Biosorption and bioaccumulationthe prospects for practical applications. Environ. Int., 36: 299-307. DOI: 10.1016/j.envint.2009.12.001

Commission, E., 2002. Directive 2002/32/EC of the European Parliament and of the Council of 7 May 2002 on undesirable substances in animal feed. Official J., 140: 10-10.

Davis, T.A., B. Volesky and A. Mucci, 2003. A review of the biochemistry of heavy metal biosorption by brown algae. Water Res., 37: 4311-4330. DOI: 10.1016/S0043-1354(03)00293-8

DPMARD, 2012. With further changes. Acceptable amounts of undesirable substances in feeds. J. Laws, 162: 11489-11494.

Dobrzanski, Z., D. Jamroz, H. Gorecka and S. Opalinski, 2003. Bioavailability of selenium and zinc supplied to the feed for laying hens in organic and inorganic form. Elect. J. Polish Agric. Univ.

Dobrzanski, Z., S. Opalinski, H. Gorecka, M. Korczyński and R. Kołacz et al., 2008. Bioavailability of $\mathrm{Fe}, \mathrm{Cu}$ and $\mathrm{Mn}$ from yeast enriched in bioelements used in laying hens feeding. Elect. J. Polish Agric. Univ.

Gupta, U.C., W. Kening and L. Siyuan, 2008. Micronutrients in soils, crops and livestock. Earth Sci. Frontiers, 15: 110-125. DOI: $10.1016 / \mathrm{S} 1872-$ 5791(09)60003-8

Hanczakowski, P., B. Szymczyk and M. Wawrzynski, 1995. Composition and nutritive value of sewagegrown duckweed (Lemna minor L.) for rats. Ani. Feed Sci. Technol., 52: 339-343. DOI: 10.1016/0377-8401(94)00729-S

Haustein, A., 1990. Duckweed, a useful strategy for feeding chickens: Performance of layers fed with sewage-grown lemnacea species. Poultry Sci., 69: 1835-1844. DOI: 10.3382/ps.0691835

Jamroz, D., 2004. Animal Nutrition and Feed Science. 1st Edn., PWN, Warszawa, Poland, pp: 89.

Johns, T. and P.B. Eyzaguirre, 2007. Biofortification, biodiversity and diet: A search for complementary applications against poverty and malnutrition. Food Policy, 32: 1-24. DOI: 10.1016/j.foodpol.2006.03.014

McDowell, L.R., 1996. Feeding minerals to cattle on pasture. Anim. Feed Sci. Technol., 60: 247-271. DOI: 10.1016/0377-8401(96)00983-2
Men, L.T., B.H. Van, M.T. Chinh and T.R. Preston, 1997. Effect of dietary protein level and duckweed (Lemna spp) on reproductive performance of pigs fed a diet of ensiled cassava root or cassava root meal. Livestock Res. Rural Devel.

Michalak, I. and K. Chojnacka, 2008. The application of macroalga Pithophora varia Wille enriched with microelements by biosorption as biological feed supplement for livestock. J. Sci. Food Agric., 88: 1178-1186. DOI: $10.1002 /$ jsfa.3195

Michalak, I. and K. Chojnacka, 2009. Edible macroalga Ulva prolifera as microelemental feed supplement for livestock: The fundamental assumptions of the production method. World J. Microbiol. Biotechnol., 25: 997-1005. DOI: 10.1007/s1 1274-009-9976-7

Michalak, I., K. Chojnacka, Z. Dobrzański, H. Gorecki and A. Zielińska et al., 2011. Effect of macroalgae enriched with microelements on egg quality parameters and mineral content of eggs, eggshell, blood, feathers and droppings. J. Ani. Physiol. Ani. Nutr., 95: 374-387. DOI: 10.1111/j.14390396.2010.01065.x

Moyo, S., J.M. Dalu and J. Ndamba, 2003. The microbiological safety of duckweed fed chickens: A risk assessment of using duckweed reared on domestic wastewater as a protein source in broiler chickens. Phys. Chem., 28: 1125-1129. DOI: 10.1016/j.pce.2003.08.021

Prasad, M.N.V., P. Malec, A. Waloszek, M. Bojko and K. Strzalka, 2001. Physiological responses of Lemna trisulca L. (duckweed) to cadmium and copper bioaccumulation. Plant Sci., 161: 881-889. DOI: 10.1016/S0168-9452(01)00478-2

Saadet, S., G. Osman, I.E. Salih and Y. Nebil, 2005. Adsorption of $\mathrm{Cd}(\mathrm{II}), \mathrm{Cu}(\mathrm{II})$ and $\mathrm{Ni}(\mathrm{II})$ ions by Lemna minor L.: Effect of physicochemical environment. J. Hazardous Mater., 126: 96-104. DOI: 10.1016/j.jhazmat.2005.06.012

Samnang, H., 1999. Duckweed versus ground soya beans as supplement for scavenging native chickens in an integrated farming system. Livestock Res. Rural Dev.

Schiewer, S. and B. Volesky, 1997. Ionic strength and electrostatic effects in biosorption of divalent metal ions and protons. Environ. Sci. Technol., 31: 2478-2485. DOI: 10.1021/es960751u

Smulikowska, S. and A. Rutkowski, 2005. Standard In Hens Feeding, 4th Edn., Instytut Fizjologii I Zywienia Zwierzat PAN, Jabłonna, Poland, pp: 36-37.

Spears, J.W., 1996. Organic trace minerals in ruminant nutrition. Anim. Feed Sci. Technol., 58: 151-163. DOI: 10.1016/0377-8401(95)00881-0

Wahaab, R.A., H.J. Lubberding and G.J. Alaerts, 1995. Copper and chromium (III) uptake by duckweed. Water Sci. Technol., 32: 105-110. DOI: 10.1016/0273-1223(96)00123-0 